\title{
Lesión diafragmática por trauma penetrante y manifestación tardía con oclusión intestinal
}

\author{
Diaphragmatic injury due to penetrating trauma and \\ late manifestation with intestinal occlusion
}

\author{
Juan Carlos Vázquez-Minero, ${ }^{*}$ Laura Portillo-Téllez, ${ }^{*}$ Jaqueline Vanessa Rodríguez-Vázquez*
}

\author{
*Central de la Cruz Roja Mexicana, Ciudad de México, México.
}

\begin{abstract}
RESUMEN. Las lesiones diafragmáticas por trauma penetrante sin manifestación aguda son poco comunes, se pueden presentar meses o años después, pero la mayoría de éstas son debidas a trauma contuso. El trauma penetrante en la mayoría de los casos da manifestaciones agudas por las lesiones a órganos abdominales o torácicos que ameritan manejo quirúrgico. Se trata de un hombre de 48 años de edad que tres años previos presentó lesiones por arma punzocortante en ambos hemitórax con manejo con sonda endopleural con buena evolución. Es egresado y reingresa por presentar dolor abdominal en epigastrio y datos de oclusión intestinal. Es estudiado y diagnosticado como hernia diafragmática postraumática izquierda con paso de estómago a cavidad torácica. Es operado con reducción del estómago y reparación del diafragma con buena evolución. En lesiones penetrantes en tórax es importante tener alto índice de sospecha en posibles lesiones de diafragma.
\end{abstract}

Palabras clave: Lesión diafragmática, hernia diafragmática postraumática, oclusión intestinal.

\section{INTRODUCCIÓN}

El trauma torácico penetrante puede presentar una mortalidad muy alta cuando afecta estructuras como corazón o grandes vasos ameritando cirugías de urgencia. Las lesiones del diafragma no ponen de manera inmediata en riesgo la vida, éstas ocurren en 3\% de todas las lesiones toracoabdominales, su frecuencia varía de 0.8 a $1.6 \%$ de los pacientes con trauma múltiple. Al no tener lesiones acompañantes pueden no ser detectadas de manera inmediata; por tanto, las lesiones inadvertidas se presentan con una frecuencia de 7 a $66 \% .^{1,2}$

\section{Correspondencia:}

\section{Dr. Juan Carlos Vázquez-Minero}

Central de la Cruz Roja Mexicana, Ciudad de México, México.

Correo electrónico: minerojc@hotmail.com

Trabajo recibido: 05-VIII-2019; aceptado: 16-X-2019.
ABSTRACT. Diaphragmatic injuries due to penetrating trauma, without acute manifestation, are rare, may occur months or years later, but most of these are due to blunt trauma. Penetrating trauma in most cases gives acute manifestations due to injuries to abdominal or thoracic organs, deserving surgical management. This is a 48-year-old man who, three years before, it was presented with stab injuries in both hemithorax with endopleural catheter management with good evolution. He is discharged from the hospital and re-enters due to abdominal pain in epigastrium and intestinal occlusion data. He is studied diagnosing left posttraumatic diaphragmatic hernia with passage from stomach to thoracic cavity. $\mathrm{He}$ was operated with reduction of the stomach and repair of the diaphragm with good evolution. In penetrating chest injuries it is important to have a high index of suspicion in possible diaphragm injuries.

Keywords: Diaphragmatic injury, posttraumatic diaphragmatic hernia, intestinal occlusion.

El diafragma presenta lesión por trauma penetrante que no es frecuente como el contuso y se puede presentar en 10 a $20 \%$ de los casos, según algunas series. ${ }^{3}$

En un estudio de nuestro Centro de Trauma de la Ciudad de México, la frecuencia de trauma penetrante fue de $6.3 \%$. En ese estudio fueron dos casos de todos los que se presentaron, los demás fueron por trauma contuso y todos fueron de presentación aguda con lesiones que ameritaron cirugía de urgencia. ${ }^{4}$

Las lesiones de presentación tardía del diafragma son más frecuentes en el trauma contuso y se relacionan con ausencia de lesiones intraabdominales como bazo, o torácicas como arcos costales o pulmón. Se pueden manifestar por sintomatología abdominal como la oclusión de manera tardía al herniarse contenido a cavidad torácica, como colon, estómago o epiplón. ${ }^{5}$

El método paraclínico de primera línea es la placa de tórax que ayuda a la sospecha diagnóstica; aunque la tomografía computarizada ayuda tanto al diagnóstico como a la planeación quirúrgica, al permitir observar el tipo de 
lesión que el diafragma presenta y su localización, además, visualizar el contenido que está herniado. ${ }^{6,7}$

El tratamiento se basa en la reducción del contenido que está herniado al tórax, la revisión de la cavidad torácica, la liberación del pulmón y la plastía del diafragma con materiales no absorbibles sintéticos a fin de evitar la posible perforación del contenido, que es una de las lesiones más graves. ${ }^{8}$

A continuación se presenta el caso de un paciente con antecedente de lesión punzocortante que da sintomatologías de oclusión tres años después de la lesión.

\section{PRESENTACIÓN DEL CASO}

Hombre de 48 años de edad que acude por presentar, tres años antes, agresión por terceras personas, con heridas por arma punzocortante en ambos hemitórax, con colocación de sondas endopleurales bilaterales, teniendo mejoría y egreso hospitalario (Figura 1). En esta ocasión ingresa por

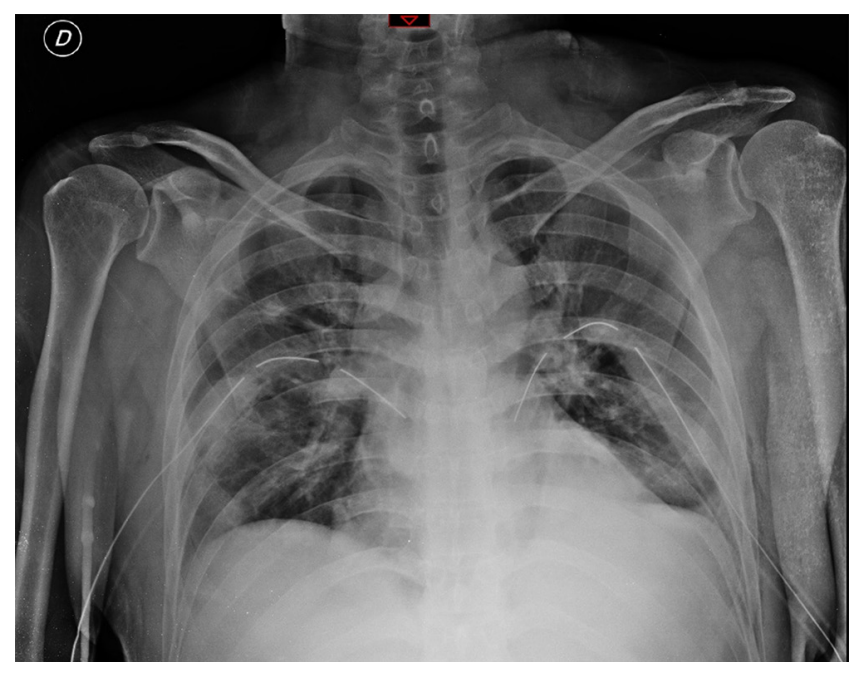

Figura 1: Radiografía de tórax con sondas pleurales bilaterales. dolor abdominal tipo cólico en epigastrio que se irradia a hipocondrio izquierdo, sin aumento ni disminución bajo ninguna circunstancia y acompañado de náusea y vómito en cuatro ocasiones de contenido alimentario, con falta de canalización de gases y evacuaciones de seis horas de evolución. EF TA 110/70 mmHg, FC 90 L/min, FR 25 R/ min, temperatura 36.8 grados. Campos pulmonares con hipoventilación basal izquierda, abdomen con peristalsis aumentada, con hiperestesia e hiperbaralgesia en epigastrio e hipocondrio izquierdo, sin datos de rebote, con timpanismo a la percusión. En la radiografía de abdomen de ingreso mostró una imagen radiolúcida que ocupa el tercio inferior del hemitórax izquierdo con ausencia de trama broncovascular. La tomografía de tórax y abdomen con trago de medio de contraste mostró un defecto en la cúpula diafragmática izquierda, por el cual hay paso de la mitad del estómago con medio de contraste en su interior, sin datos de compromiso de vísceras abdominales (Figuras $2 A$ y $B$ ). Ingresó a quirófano y se hizo el abordaje por toracotomía posterolateral izquierda, se apreció el estómago herniado hacia la cavidad torácica; en el procedimiento el estómago presentó adherencias al pulmón, las cuales se despegaron y se redujo hacia la cavidad abdominal a través de un defecto del diafragma de $5 \mathrm{~cm}$. Se cerró el defecto diafragmático a dos planos con puntos de colchonero con prolene del 1 (Figuras 3A-C), se dejó una sonda endopleural No. 32 que se retiró al segundo día. El paciente inició dieta líquida a las 24 horas de operado y posteriormente blanda, se egresó del hospital al cuarto día de la cirugía con cita a consulta un mes después con adecuada evolución.

\section{DISCUSIÓN}

El trauma torácico representa uno de cada cuatro pacientes lesionados por múltiples mecanismos. Las lesiones más difíciles de diagnosticar son las diafragmáticas, éstas ocurren en el 3\% de todas las lesiones toracoabdominales, su frecuencia
Figura 2:

A) Radiografía de tórax con estómago en la cavidad torácica. B) Tomografía Axial Computarizada del defecto diafragmático.
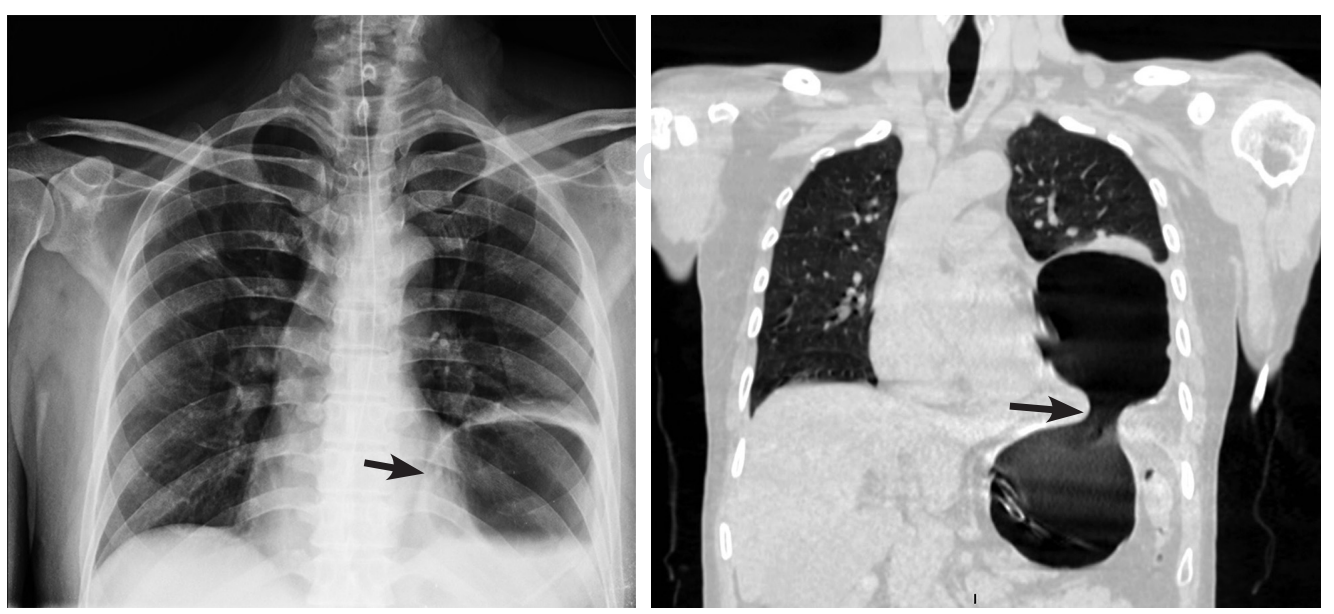

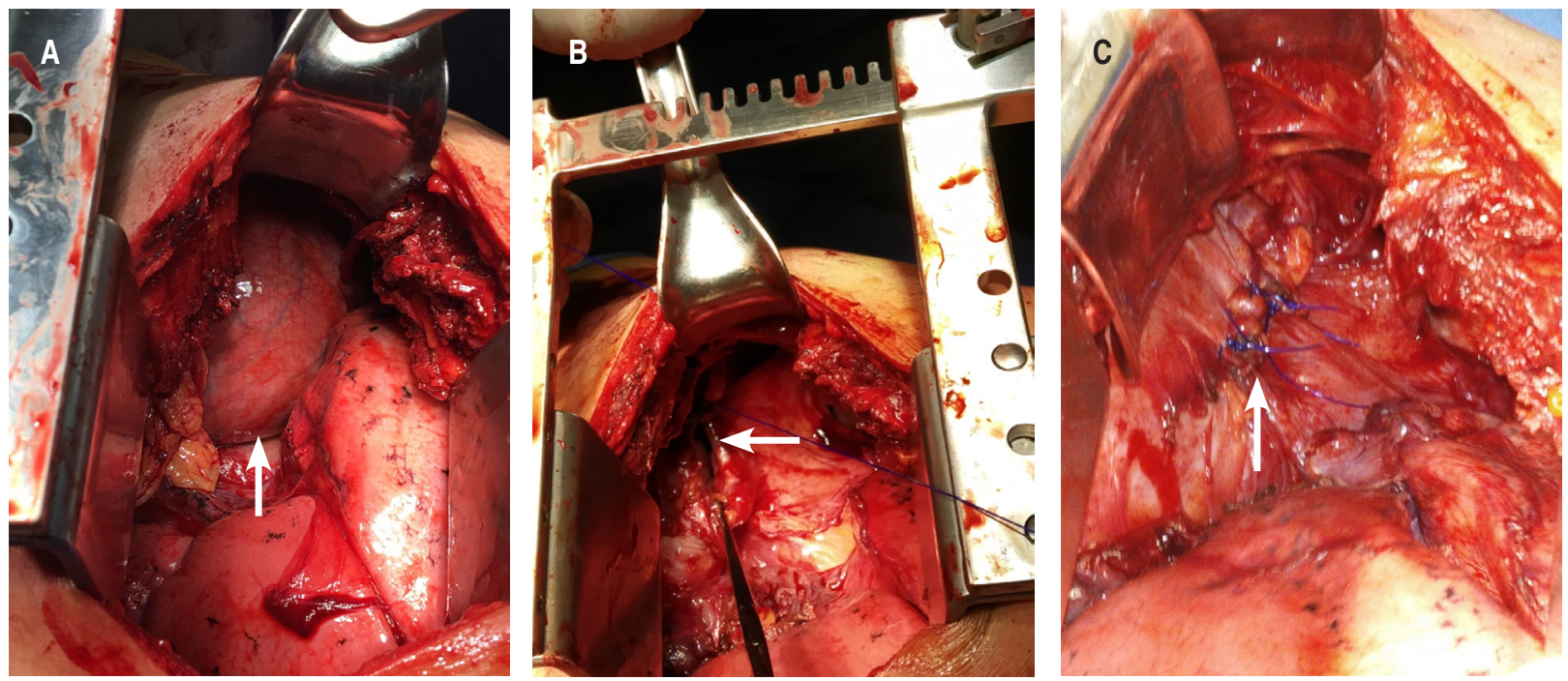

Figura 3: A) Estómago en cavidad torácica. B) Defecto diafragmático. C) Cierre del defecto.

varía de 0.8 a $1.6 \%$ de los pacientes con trauma múltiple. Pueden no ser detectadas de manera inmediata; por tanto, las lesiones inadvertidas se presentan con una frecuencia de 7 a $66 \%{ }^{1,2}$ El diafragma presenta lesión por trauma penetrante en 10 a 20\% de los casos. Éste es el primer caso en los últimos 14 años que se presenta en el hospital. ${ }^{3}$

En un estudio de nuestro Centro de Trauma de la Ciudad de México, la frecuencia de trauma penetrante fue de 6.3\%, en ese estudio fueron dos casos de presentación aguda, el nuestro fue de presentación tardía que es mucho más raro. ${ }^{4}$

Las lesiones de presentación tardía del diafragma son más frecuentes en el trauma contuso y se relacionan con ausencia de lesiones intraabdominales o torácicas. Se pueden manifestar por sintomatología abdominal como la oclusión. En este caso no existió como tal lesión intraabdominal, solamente la herniación del estómago a la cavidad torácica y su encarceramiento con sintomatología de oclusión intestinal. ${ }^{5}$

El método paraclínico que ayuda, tanto al diagnóstico como a la planeación quirúrgica, es la tomografía computarizada toracoabdominal. En nuestro caso nos ayudó al diagnóstico y a la planeación del abordaje quirúrgico a través del tórax. ${ }^{6,7}$ El tratamiento se basa en la reducción del estómago y la plastía del diafragma como en nuestro caso, a fin de evitar la posible perforación del estómago que es una de las lesiones más graves. ${ }^{8}$

\section{CONCLUSIONES}

Las lesiones de diafragma agudas que no presentan lesiones asociadas pueden pasar inadvertidas hasta dar manifes- taciones clínicas abdominales o torácicas que ameritan el estudio del paciente. Por tanto, toda lesión contusa o penetrante debe de tener un seguimiento para evitar complicaciones.

\section{REFERENCIAS}

1. Ward RE, Flynn TC, Clark WP. Diaphragmatic disruption secondary to blunt abdominal trauma. J Trauma 1981;21:35-38. https://doi. org/10.1097/00005373-198101000-00006

2. Mansour KA. Trauma to the diaphragm. Chest Surg Clin N Am 1997;7(2):373-383.

3. Adegboye VO, Ladipo JK, Adebo OA, Brimmo Al. Diaphragmatic injuries. Afr J Med Med Sci 2002;31(2):149-153.

4. Ferrufino MAL, Vázquez MJC, Delgadillo GS. Lesiones diafragmáticas por trauma contuso. Experiencia en un centro de trauma. Cir Gen 2009;31(1):26-30.

5. Vázquez MJC, Arriola NJM, Quiroga AVE, Cervantes SY. Ruptura diafragmática postraumática de presentación tardía. A propósito de dos casos. Neumol Cir Torax 2018;77(2):151-156.

6. Bosanquet D, Farboud A, Luckraz H. A review diaphragmatic injury. Respiratory Medicine CME 2009;2(1):1-6. https://dx.doi.org/10.1016/j. rmedc.2009.01.002

7. Olivares-Becerra JJ, Farías-Llamas OA, Candelas-del Toro 0 , Medrano-Muñoz F, Fuentes-Orozco C, González-Ojeda A. Hernia diafragmática traumática. Cir Ciruj 2006;74(6):415-423.

8. Carranza-Sarmina J, Ríos-Pascual S, Vázquez-Minero JC. Perforación gástrica secundaria a hernia congénita de Bochdaleck en un adolescente. Reporte de un caso. Neumol Cir Torax 2018;77(1):24-27.

Conflicto de intereses: Los autores declaran no tener conflicto de intereses. 